\title{
Black-Scholes option pricing within Itô and Stratonovich conventions
}

\author{
J. Perellóa ${ }^{\mathrm{a}}$, J. M. Porrà ${ }^{\mathrm{a}, \mathrm{b}}$, M. Montero ${ }^{\mathrm{a}}$ and J. Masoliver ${ }^{\mathrm{a}}$ \\ ${ }^{a}$ Departament de Física Fonamental, Universitat de Barcelona, Diagonal, 647, \\ 08028-Barcelona, Spain \\ ${ }^{\mathrm{b}}$ Gaesco Bolsa, SVB, S.A., Diagonal, 429, 08036-Barcelona, Spain
}

\begin{abstract}
Options are financial instruments designed to protect investors from the stock market randomness. In 1973, Fisher Black, Myron Scholes and Robert Merton proposed a very popular option pricing method using stochastic differential equations within the Itô interpretation. Herein, we derive the Black-Scholes equation for the option price using the Stratonovich calculus along with a comprehensive review, aimed to physicists, of the classical option pricing method based on the Itô calculus. We show, as can be expected, that the Black-Scholes equation is independent of the interpretation chosen. We nonetheless point out the many subtleties underlying Black-Scholes option pricing method.
\end{abstract}

\section{Introduction}

An European option is a financial instrument giving to its owner the right but not the obligation to buy (European call) or to sell (European put) a share at a fixed future date, the maturing time $T$, and at a certain price called exercise or striking price $x_{C}$. In fact, this is the most simple of a large variety of contracts that can be more sophisticated. One of those possible extensions is the American option which gives the right to exercise the option at any time until the maturing time. In a certain sense, options are a security for the investor thus avoiding the unpredictable consequences of operating with risky speculative stocks.

The trading of options and their theoretical study have been known for long, although they were relative obscure and unimportant financial instruments until the early seventies. It was then when options experimented an spectacular development. The Chicago Board Options Exchange, created in 1973, is the first attempt to unify options in one market and trade them on only a few 
stock shares. The market rapidly became a tremendous success and led to a series of innovations in option trading [1].

The main purpose in option studies is to find a fair and presumably riskless price for these instruments. The first solution to the problem was given by Bachelier in 1900 [2], and several option prices were proposed without being completely satisfactory [3]. However, in the early seventies it was finally developed a complete option valuation based on equilibrium theoretical hypothesis for speculative prices. The works of Fisher Black, Myron Scholes [4] and Robert Merton [5] were the culmination of this great effort, and left the doors open for extending the option pricing theory in many ways. In addition, the method has been proved to be very useful for investors and has helped to option markets to have the importance that they have nowadays in finance $[1,3]$.

The option pricing method obtains the so-called Black-Scholes equation which is a partial differential equation of the same kind as the diffusion equation. In fact, it was this similarity that led Black and Scholes to obtain their option price formula as the solution of the diffusion equation with the initial and boundary conditions given by the option contract terms. Incidentally, these physics studies applied to economy have never been disrupted and there still is a growing effort of the physics community to understand the dynamics of finance from approaches similar to those that tackle complex systems in physics [6-10].

The economic ideas behind the Black-Scholes option pricing theory translated to the stochastic methods concepts are as follows. First, the option price depends on the stock price and this is a random variable evolving with time. Second, the efficient market hypothesis [11], i.e., the market incorporates instantaneously any information concerning future market evolution, implies that the random term in the stochastic equation must be delta-correlated. That is: speculative prices are driven by white noise $[6,12]$. It is known that any white noise can be written as a combination of the derivative of the Wiener process and white shot noise [13]. In this framework, the Black-Scholes option pricing method was first based on the geometric Brownian motion [4,5], and it was lately extended to include white shot noise $[14,15]$.

As is well known, any stochastic differential equation (SDE) driven by a state dependent white noise, such as the geometric Brownian motion, is meaningless unless an interpretation of the multiplicative noise term is given. Two interpretations have been presented: Itô [16] and Stratonovich [17]. To our knowledge, all derivations of the Black-Scholes equation starting from a SDE are based on the Itô interpretation. A possible reason is that mathematicians prefer this interpretation over the Stratonovich's one, being the latter mostly preferred among physicists. Nonetheless, as we try to point out here, Itô framework is 
perhaps more convenient for finance being this basically due to the peculiarities of trading (see Sect. 4). In any case, as Van Kampen showed some time ago [18] no physical reason can be attached to the interpretation of the SDE modelling price dynamics. However, the same physical process results in two different SDEs depending on the interpretation chosen. In spite of having different differential equations as starting point, we will show that the resulting Black-Scholes equation is the same regardless the interpretation of the multiplicative noise term, and this constitutes the main result of the paper. In addition, the mathematical exercise that represents this translation into the Stratonovich convention provides a useful review, specially to physicists, of the option pricing theory and the "path-breaking" Black-Scholes method.

The paper is divided in 5 sections. After the Introduction, a summary of the differences between Itô and Stratonovich calculus is developed in Section 2. The following section is devoted to explain the market model assumed in Black-Scholes option pricing method. Section 4 concentrates in the derivation of the Black-Scholes equation using both Itô and Stratonovich calculus. Conclusions are drawn in Section 5, and some technical details are left to appendices.

\section{Itô vs. Stratonovich}

It is not our intention to write a formal discussion on the differences between Itô and Stratonovich interpretations of stochastic differential equations since there are many excellent books and reviews on the subject [13,18-20]. However, we will summarize those elements in these interpretations that change the treatment of the Black-Scholes option pricing method. In all our discussion, we use a notation that it is widely used among physicists.

The interpretation question arises when dealing with a multiplicative stochastic differential equation, also called multiplicative Langevin equation,

$$
\dot{X}=f(X)+g(X) \xi(t)
$$

where $f$ and $g$ are given functions, and $\xi(t)$ is Gaussian white noise, that is, a Gaussian and stationary random process with zero mean and delta correlated. Alternatively, Eq. (1) can be written in terms of the Wiener process $W(t)$ as

$$
d X=f(X) d t+g(X) d W(t)
$$

where $d W(t)=\xi(t) d t$. When $g$ depends on $X$, Eqs. (1) and (2) have no meaning, unless an interpretation of the multiplicative term $g(X) \xi(t)$ is provided. 
These different interpretations of the multiplicative term must be given because, due to the extreme randomness of white noise, it is not clear what value of $X$ should be used even during an infinitesimal timestep $d t$. According to Itô, that value of $X$ is the one before the beginning of the timestep, i.e., $X=X(t)$, whereas Stratonovich uses the value of $X$ at the middle of the timestep: $X=X(t+d t / 2)=X(t)+d X(t) / 2$.

Before proceeding further with the consequences of the above discussion, we will first give a precise meaning of the differential of random processes driven by Gaussian white noise and its implications. Obviously, the differential of any random process $X(t)$ is defined by

$$
d X(t) \equiv X(t+d t)-X(t)
$$

On the other hand, the differential $d X(t)$ of any random process is equal (in the mean square sense) to its mean value if its variance is, at least, of order $d t^{2}[13]:\left\langle[d X(t)-\langle d X(t)\rangle]^{2}\right\rangle=O\left(d t^{2}\right)$. We observe that from now on all the results of this paper must be interpreted in the mean square sense. The mean square limit relation can be used to show that $|d W(t)|^{2}=d t[20]$. We thus have from Eq. (2) that

$$
|d X|^{2}=|g(X)|^{2} d t+O\left(d t^{2}\right)
$$

and we symbolically write

$$
d X(t)=O\left(d t^{1 / 2}\right)
$$

Let us now turn our attention to the differential of the product of two random processes since this differential adopts a different expression depending on the interpretation (Itô or Stratonovich) chosen. In accordance to Eq. (3), we define

$$
d(X Y) \equiv[(X+d X)(Y+d Y)]-X Y
$$

This expression can be rewritten in many different ways. One possibility is

$$
d(X Y)=\left(X+\frac{d X}{2}\right) d Y+\left(Y+\frac{d Y}{2}\right) d X
$$

but it is also allowed to write the product as

$$
d(X Y)=X d Y+Y d X+d X d Y
$$


Therefore, we say that the differential of a product reads in the Stratonovich interpretation when

$$
d(X Y) \equiv X_{S} d Y+Y_{S} d X
$$

where

$$
X_{S}(t) \equiv X(t+d t / 2)=X(t)+d X(t) / 2
$$

and similarly for $Y_{S}(t)$. Whereas we say that the differential of a product follows the Itô interpretation when

$$
d(X Y) \equiv X_{I} d Y+Y_{I} d X+d X d Y
$$

where

$$
X_{I}(t) \equiv X(t)
$$

and $Y_{I}(t) \equiv Y(t)$. Note that Eq. (9) formally agrees with the rules of calculus while Eq. (11) does not. Note also that Eqs. (9) and (11) can easily be generalized to the product of two functions, $U(X)$ and $V(X)$, of the random process $X=X(t)$. Thus

$$
d(U V)=U\left(X_{S}\right) d V(X)+V\left(X_{S}\right) d U(X),
$$

where $X_{S}$ is given by Eq. (10), and $d V(X)=V(X+d X)-V(X)$ with an analogous expression for $d U(X)$. Within Itô convention we have

$$
d(U V)=U(X) d V(X)+V(X) d U(X)+d U(X) d V(X) .
$$

Let us now go back to Eq. (1) and see that one important consequence of the above discussion is that the expected value of the multiplicative term, $g(X) \xi(t)$, depends on the interpretation given. In the Itô interpretation, it is clear that $\langle g(X) \xi(t)\rangle=0$ because the value of $X$ (and, hence the value of $g(X))$ anticipates the jump in the noise. In other words, $g(X)$ is independent of $\xi(t)$. On the other hand, it can be proved that within the Stratonovich framework the average of the multiplicative term reads $g(X) g^{\prime}(X) / 2$ where the prime denotes the derivative [20]. The zero value of the average $\langle g(X) \xi(t)\rangle$ makes Itô convention very appealing because then the deterministic equation for the mean value of $X$ only depends on the drift term $f(X)$. In this sense, note that any multiplicative stochastic differential equation has different expressions for the functions $f(X)$ and $g(X)$ depending on the interpretation 
chosen. In the Stratonovich framework, a SDE of type Eq. (2) can be written as

$$
d X=f^{(S)}\left(X_{S}\right) d t+g^{(S)}\left(X_{S}\right) d W(t)
$$

where $X_{S}=X+d X / 2$. In the Itô sense we have

$$
d X=f^{(I)}\left(X_{I}\right) d t+g^{(I)}\left(X_{I}\right) d W(t)
$$

where $X_{I}=X$. Note that $f^{(S)}$ and $f^{(I)}$ are not only evaluated at different values of $X$ but are also different functions depending on the interpretation given, and the same applies to $g^{(S)}$ and $g^{(I)}$. One can easily show from Eq. (10) and Eqs. (15)-(16) that, after keeping terms up to order $d t$, the relation between $f_{S}$ and $f_{I}$ is $[20]$

$$
f^{(I)}(X)=f^{(S)}(X)-\frac{1}{2} g^{(S)}(X) \frac{\partial g^{(S)}(X)}{\partial X}
$$

while the multiplicative functions $g^{(S)}$ and $g^{(I)}$ are equal

$$
g^{(I)}(X)=g^{(S)}(X)
$$

Conversely, it is possible to pass from a Stratonovich SDE to an equivalent Itô SDE [20]. Note that the difference between both interpretation only affects the drift term given by the function $f$ while the function $g$ remains unaffected. In addition, we see that for an additive SDE, i.e., when $g$ is independent of $X$, the interpretation question is irrelevant.

Finally, a crucial difference between Itô and Stratonovich interpretations appears when a change of variables is performed on the original equation. Then it can be proved that, using Stratonovich convention, the standard rules of calculus hold, but new rules appear when the equation is understood in the Itô sense. From the point of view of this property, the Stratonovich criterion seems to be more convenient. For the sake of completeness, we remind here what are the rules of change of variables in each interpretation. Let $h(X, t)$ be an arbitrary function of $X$ and $t$. In the Itô sense, the differential of $h(X, t)$ reads [20]

$$
d h=\frac{\partial h(X, t)}{\partial X} d X+\left[\frac{\partial h(X, t)}{\partial t}+\frac{1}{2} g^{2}(X, t) \frac{\partial^{2} h(X, t)}{\partial X^{2}}\right] d t
$$


whereas in the Stratonovich sense, we have the usual expression [20]

$$
d h=\frac{\partial h\left(X_{S}, t\right)}{\partial X_{S}} d X+\frac{\partial h\left(X_{S}, t\right)}{\partial t} d t
$$

where

$$
\frac{\partial h\left(X_{S}, t\right)}{\partial X_{S}}=\left.\frac{\partial h(X, t)}{\partial X}\right|_{X=X_{S}},
$$

and $X_{S}$ is given by Eq. (10).

Equation (19) is known as the Itô's lema and it is extensively used in mathematical finance books $[12,21-25]$.

The information on the properties of the Itô and Stratonovich interpretation of SDE contained in this brief summary is sufficient to follow the derivations of the next sections.

\section{Market model}

Option pricing becomes a problem because market prices or indexes change randomly. Therefore, any possible calculation of an option price is based on a model for the stochastic evolution of the market prices. The first analysis of price changes was given one hundred years ago by Bachelier who, studying the option pricing problem, proposed a model assuming that price changes behave as an ordinary random walk [2]. Thus, in the continuum limit (continous time finance [25]) speculative prices obey a Langevin equation. In order to include the limited liability of the stock prices, i.e., prices cannot be negative, Osborne proposed the geometric or log-Brownian motion for describing the price changes [26]. Mathematically, the market model assumed by Osborne can be written as a stochastic equation of type Eq. (2):

$$
d R(t)=\mu d t+\sigma d W(t)
$$

where $R(t)$ is the so-called return rate after a period $t$. Therefore, $d R(t)$ is the infinitessimal relative change in the stock share price $X(t)$ (see Eq. (22) below), $\mu$ is the average rate per unit time, and $\sigma^{2}$ is the volatility per unit time of the rate after a period $t$, i.e., $\langle d R\rangle=\mu d t$ and $\left\langle(d R-\langle d R\rangle)^{2}\right\rangle=\sigma^{2} d t$. There is no need to specify an interpretation (Itô's or Stratonovich's) for Eq. (21) because $\sigma$ is constant and we are thus dealing with an additive equation. The rate is compounded continuously and, therefore, an initial price $X_{0}$ becomes after a period $t$ :

$$
X(t)=X_{0} \exp [R(t)]
$$


This equation can be used as a change of variables to derive the SDE for $X(t)$ given that $R(t)$ evolves according to Eq. (21). However, as it becomes multiplicative, we have to attach the equation to an interpretation. Indeed, using Stratonovich calculus (see Eq. (20)), it follows that $X(t)$ evolves according to the equation

$$
d X=\mu X_{S} d t+\sigma X_{S} d W(t)
$$

where $X_{S}=X+d X / 2$. In the Itô sense (see Eq. (19)), the equation for $X(t)$ becomes

$$
d X=\left(\mu+\sigma^{2} / 2\right) X d t+\sigma X d W(t) .
$$

Therefore, the Langevin equation for $X(t)$ is different depending on the sense it is interpreted. The main objective of this paper is to show that no matter which equation is used to derive the Black-Scholes equation the final result turns out to be the same.

Before proceeding further, we point out that the average index price after a time $t$ is $\langle X(t)\rangle=X_{0} \exp \left(\mu+\sigma^{2} / 2\right) t$, regardless the convention being used. In fact, the independence of the averages on the interpretation used holds for moments of any order [18-20].

\section{The Black-Scholes equation}

There are several different approaches for deriving the Black-Scholes equation starting from the stochastic differential equation point of view. These different derivations only differ in the way the portfolio (i.e., a collection of different assets for diversifying away financial risk) is defined $[4,25,27,28]$. In order to get the most general description of the concepts underlying in the Black-Scholes theory, our portfolio is similar to the one proposed by Merton [27], and it is based on one type of share whose price is the random process $X(t)$. The portfolio is compounded by a certain amount of shares, $\Delta$, a number of calls, $\Psi$, and, finally, a quantity of riskless securities (or bonds) $\Phi$. We also assume that short-selling, or borrowing, is allowed. Specifically, we own a certain number of calls worth $\Psi C$ dollars and we owe $\Delta X+\Phi B$ dollars. In this case, the value $P$ of the porfolio reads

$$
P=\Psi C-\Delta X-\Phi B,
$$

where $X$ is the share stock price, $C$ is the call price to be determined, and $B$ is the bond price whose evolution is not random and is described according to 
the value of $r$, the risk-free interest rate ratio. That is

$$
d B=r B d t
$$

The so-called "portfolio investor's strategy" [22] decides the quantity to be invested in every asset according to its stock price at time $t$. This is the reason why the asset amounts $\Delta, \Psi$, and $\Phi$ are functions of stock price and time, although they are "nonanticipating" functions of the stock price. This somewhat obscure concept is explained in the Appendix A. All derivations of Black-Scholes equation assume a "frictionless market", that is, there are no transaction costs for each operation of buying and selling [4].

According to Merton [27] we assume that, by short-sales, or borrowing, the portfolio (25) is constrained to require net zero investment, that is, $P=0$ for any time $t$ [29]. Then, from Eq. (25) we have

$$
C=\delta_{n} X+\phi_{n} B
$$

where, $\delta_{n} \equiv \Delta / \Psi$ and $\phi_{n} \equiv \Phi / \Psi$ are respectively the number of shares per call and the number of bonds per call. As we have mentioned above, $\delta_{n}$ and $\phi_{n}$ are nonanticipating functions of the stock price (see Appendix A). Note that Eq. (27) has an interesting economic meaning, since tells us that having a call option is equivalent to possess a certain number, $\delta_{n}$ and $\phi_{n}$, of shares and bonds thus avoiding any arbitrage opportunity [29]. Equation (27), which is called "the replicating portfolio" $[12,22,23]$, is the starting point of our derivation that we separate into two subsections according to Itô or Stratonovich interpretations.

\subsection{The Black-Scholes equation derivation (Itô)}

We need first to obtain, within the Itô interpretation, the differential of the call price $C$. This is done in the Appendix B and we show there that

$$
d C=\delta d X+\phi d B+X d \delta_{n}+B d \phi_{n}+O\left(d t^{3 / 2}\right),
$$

where the relationship between $\delta, \phi$ and $\delta_{n}, \phi_{n}$ is given in Appendix A ( $c f$. Eq. (A.1)). We assume we follow a "self-financing strategy" [28], that is, variations of wealth are only due to capital gains and not to the withdrawal or infusion of new funds. In other words, we increase [decrease] the number of shares by selling [buying] bonds in the same proportion. We then have (see Appendix A for more details)

$$
X d \delta_{n}=-B d \phi_{n}
$$


and Eq. (28) reads

$$
d C=\delta d X+\phi d B .
$$

Moreover, from Eqs. (26)-(27) one can easily show that

$$
\phi d B=r(C-\delta X) d t+O\left(d t^{3 / 2}\right),
$$

(cf. Eq. (5) and Eq. (A.1) of Appendix A). Therefore,

$$
d C=\delta d X+r(C-\delta X) d t+O\left(d t^{3 / 2}\right) .
$$

On the other hand, since the call price $C$ is a function of share price $X$ and time, $C=C(X, t)$, and $X$ obeys the (Itô) SDE (24), then $d C$ can be evaluated from the Itô lemma (19) with the result

$$
d C=\left(\frac{\partial C}{\partial t}+\frac{1}{2} \sigma^{2} X^{2} \frac{\partial^{2} C}{\partial X^{2}}\right) d t+\frac{\partial C}{\partial X} d X .
$$

Substituting Eq. (31) into Eq. (32) yields

$$
\left(\delta-\frac{\partial C}{\partial X}\right) d X=\left[\frac{\partial C}{\partial t}-r(C-\delta X)+\frac{1}{2} \sigma^{2} X^{2} \frac{\partial^{2} C}{\partial X^{2}}\right] d t .
$$

Note that this is an stochastic equation because of its dependence on the Wiener process enclosed in $d X$. We can thus turn Eq. (33) into a deterministic equation that will give the call price functional dependence on share price and time by equating to zero the term multiplying $d X$. This, in turn, will determine the "investor strategy", that is the number of shares per call, the so called "delta hedging":

$$
\delta=\frac{\partial C(x, t)}{\partial x} .
$$

The substitution of Eq. (34) into Eq. (33) results in the Black-Scholes equation:

$$
\frac{\partial C}{\partial t}=r C-r x \frac{\partial C}{\partial x}-\frac{1}{2}(\sigma x)^{2} \frac{\partial^{2} C}{\partial x^{2}} .
$$

A final observation, in Eqs. (34)-(35) we have set $X=x$, since, as explained above, Eq. (35) gives the functional dependence of the call price $C$ on $X$ and $t$ regardless whether the share price $X$ is random or not. 


\subsection{The Black-Scholes equation derivation (Stratonovich)}

Let us now derive the Black-Scholes equation, assuming that the underlying asset obeys the Stratonovich SDE (23). In the Appendix B we present part of this derivation using the concept of nonanticipating function within the Stratonovich interpretation. Nevertheless, here we perform an alternative derivation that uses the Itô interpretation as starting point. We thus begin with Eq. (31) that we write in the form

$$
d C=\delta(X, t) d X(t)+r[C(X, t)-\delta(X, t) X] d t+O\left(d t^{3 / 2}\right) .
$$

Now, we have to express the function $\delta$ within Stratonovich interpretation. Note that $X=X_{S}-d X / 2$. Hence $\delta(X, t)=\delta\left(X_{S}-d X / 2, t\right)$, whence

$$
\delta(X, t)=\delta\left(X_{S}, t\right)-\frac{1}{2} \frac{\partial \delta\left(X_{S}, t\right)}{\partial X_{S}} d X+O\left(d X^{2}\right) .
$$

Analogously $C(X, t)=C\left(X_{S}, t\right)+O(d X)$. Therefore, from Eqs. (36)-(37) and taking into account Eq. (4) we have

$$
\begin{aligned}
d C=\delta\left(X_{S}, t\right) d X+\left[r C\left(X_{S}, t\right)\right. & -r X_{S} \delta\left(X_{S}, t\right) \\
& \left.-\frac{1}{2} \sigma^{2} X_{S}^{2} \frac{\partial \delta\left(X_{S}, t\right)}{\partial X_{S}}\right] d t+O\left(d t^{3 / 2}\right) .
\end{aligned}
$$

On the other hand, $d C$ will also be given by Eq. (20)

$$
d C=\frac{\partial C\left(X_{S}, t\right)}{\partial t} d t+\frac{\partial C\left(X_{S}, t\right)}{\partial X_{S}} d X,
$$

From these two equations we get

$$
\begin{aligned}
{\left[\delta\left(X_{S}, t\right)-\frac{\partial C\left(X_{S}, t\right)}{\partial X_{S}}\right] d X } & =\left[\frac{\partial C\left(X_{S}, t\right)}{\partial t}-r C\left(X_{S}, t\right)\right. \\
& \left.+r X_{S} \delta\left(X_{S}, t\right)+\frac{1}{2} \sigma^{2} X_{S}^{2} \frac{\partial \delta\left(X_{S}, t\right)}{\partial X_{S}}\right] d t .
\end{aligned}
$$

Again, this equation becomes non-stochastic if we set

$$
\delta\left(X_{S}, t\right)=\frac{\partial C\left(X_{S}, t\right)}{\partial X_{S}} .
$$


In this case, the combination of Eqs. (40)-(41) agrees with Eq. (35). Although the call price is evaluated at a different value of the share price, this is irrelevant for the reason explained right after Eq. (35). Therefore, the Stratonovich calculus results in the same call price formula and equation than the Itô calculus.

We have used the stochastic differential equation technique in order to derive the option price equation. However, this is only one of the possible routes. Another way, which was also proposed in the original paper of Black and Scholes [4], uses the Capital Asset Pricing Model (CAPM) [30] where, adducing equilibrium reasons in the asset prices, it is assumed the equality of the socalled "Sharpe ratio" of the stock and the option respectively. The Sharpe ratio of an asset can be defined as its normalized excess of return, therefore CAPM assumption applied to option pricing reads [25]

$$
\frac{\alpha-r}{\sigma}=\frac{\alpha_{C}-r}{\sigma_{C}}
$$

where $\alpha=\langle d X / X\rangle, \sigma^{2}=\operatorname{Var}(d X / X), \alpha_{C}=\langle d C / C\rangle$, and $\sigma_{C}^{2}=\operatorname{Var}(d C / C)$. From this equality it is quite straightforward to derive the Black-Scholes equation $[4,25]$. As remarked at the end of Sect. 3, moments are independent of the interpretation chosen, we thus clearly see the equivalence between Itô and Stratonovich calculus for the Black-Scholes equation derivation.

\subsection{The Black-Scholes formula for the European call}

For the sake of completeness, let us now finish the paper by shortly deriving from Eq. (35) the well-known Black-Scholes formula. Note that the BlackScholes equation is a backward parabolic differential equation, we therefore need one "final" condition and, in principle, two boundary conditions in order to solve it [31]. In fact, Black-Scholes equation is defined on the semi-infinite interval $0 \leq x<\infty$. In this case, since $C(x, t)$ is assumed to be sufficiently well behaved for all $x$, we only need to specify one boundary condition at $x=0$ (see [24] and [31]), although we specify below the boundary condition at $x=\infty$ as well.

We also note that all financial derivatives (options of any kind, forwards, futures, swaps, etc...) have the same boundary conditions but different initial or final condition [23]. Let us first specify the boundary conditions. We see from the multiplicative character of Eq. (2) that if at some time the price $X(t)$ drops to zero then it stays there forever. In such a case, it is quite obvious that the call option is worthless:

$$
C(0, t)=0
$$


On the other hand, as the share price increases without bound, $X \rightarrow \infty$, the difference between share price and option price vanishes, since option is more and more likely to be exercised and the value of the option will agree with the share price, that is,

$$
\lim _{x \rightarrow \infty} \frac{C(x, t)}{x}=1
$$

In order to obtain the "final" condition for Eq. (35), we need to specify the following two parameters: the expiration or maturing time $T$, and the striking or exercise price $x_{C}$ that fixes the price at which the call owner has the right to buy the share at time $T$. If we want to avoid arbitrage opportunities, it is clear that the value of the option $C$ of a share that at time $T$ is worth $x$ dollars must be equal to the payoff for having the option [2]. This payoff is either 0 or the difference between share price at time $T$ and option striking price, that is, $\max \left(x-x_{C}, 0\right)$. Hence, the "final" condition for the European call is

$$
C(x, t=T)=\max \left(x-x_{C}, 0\right) .
$$

In the Appendix $\mathrm{C}$ we show that the solution to the problem given by Eq. (35) and Eqs. (42)-(44) is

$$
C(x, t)=x N\left(d_{1}\right)-x_{C} e^{-r(T-t)} N\left(d_{2}\right),
$$

$(0 \leq t \leq T)$, where

$$
N(z)=\frac{1}{\sqrt{2 \pi}} \int_{-\infty}^{z} e^{-u^{2} / 2} d u
$$

is the probability integral,

$$
d_{1}=\frac{\ln \left(x / x_{c}\right)+\left(r+\sigma^{2} / 2\right)(T-t)}{\sigma \sqrt{T-t}},
$$

and

$$
d_{2}=d_{1}-\sigma \sqrt{T-t} .
$$

\section{Conclusions}

We have updated the option pricing theory from the point of view of a physicist. We have centered our analysis of option pricing to the Black-Scholes equation and formula for the European call, extensions to other kind of options can be straightforward in many cases and are found in several good 
finance books [21-25]. We have reviewed Black-Scholes theory using Itô calculus, which is standard to mathematical finance, with a special emphasis in explaining and clarifying the many subtleties of the calculation. Nevertheless, we have not limit ourselves only to review option pricing, but to derive, for the first time to our knowledge, the Black-Scholes equation using the Stratonovich calculus which is standard to physics, thus bridging the gap between mathematical finance and physics.

As we have proved, the Black-Scholes equation obtained using Stratonovich calculus is the same as the one obtained by means of the Itô calculus. In fact, this is the result we expected in advance because Itô and Stratonovich conventions are just different rules of calculus. Moreover, from a practical point of view, both interpretations differ only in the drift term of the Langevin equation and the drift term does not appear in the Black-Scholes equation and formula. But, again, we think that this derivation is still interesting and useful for all the reasons explained above.

\section{Acknowledgements}

This work has been supported in part by Dirección General de Investigación Científica y Técnica under contract No. PB96-0188 and Project No. HB1190104, and by Generalitat de Catalunya under contract No. 1998 SGR-00015.

\section{A Nonanticipating functions and self-financing strategy}

The functionals $\phi_{n}$ and $\delta_{n}$ representing normalized asset quantities are nonaticipating functions with respect to the stock price $X$. This means that these functionals are in some way independent of $X(t)$ implying a sort of causality in the sense that unknown future stock price cannot affect the present portfolio strategy. The physical meaning of this translated to financial markets is: first buy or sell according to the present stock price $X(t)$ and right after the portfolio worth changes with variation of the prices $d X, d B$, and $d C$. In other words, the investor strategy does not anticipate the stock price change [3,23]. Therefore, in the Itô sense, the functionals $\delta_{n}$ and $\phi_{n}$ representing the number of assets in the portfolio solely depend on the share price right before time $t$, i.e., they do not depend on $X(t)$ but on $X(t-d t)=X-d X$. That is,

$$
\delta_{n}(X, t) \equiv \delta(X-d X, t)
$$


and similarly for $\phi_{n}$ (recall that all equalities must be understood in the mean square sense explained in Sect. 2).

The expansion of Eq. (A.1) yields (see Eq. (5))

$$
\delta_{n}(X, t)=\delta(X, t)-\frac{\partial \delta(X, t)}{\partial X} d X+O(d t)
$$

but from the Itô lema (19) we see that

$$
\frac{\partial \delta(X, t)}{\partial X} d X=d \delta(X, t)+O(d t)
$$

and finally

$$
\delta_{n}(X, t)=\delta(X, t)-d \delta(X, t)+O(d t)
$$

Analogously,

$$
\delta(X, t)=\delta_{n}(X, t)+d \delta_{n}(X, t)+O(d t)
$$

and a similar expresion for $\phi(X, t)$.

As to the self-financing strategy, Eq. (29), we observe that $\delta(X, t+d t)$ is the number of shares we have at time $t+d t$, while $\delta(X-d X, t)$ is that number at time $t$. Therefore,

$$
X(t) d \delta(X-d X, t)=[\delta(X, t+d t)-\delta(X-d X, t)] X(t)
$$

is the money we need or obtain for buying or from selling shares at time $t$. Analogously, $B(t) d \phi(X-d X, t)$ is the money, needed or obtained at time $t$, coming from bonds. If we follow a self-financing strategy, both quantities are equal but with different sign, i.e.,

$$
X(t) d \delta(X-d X, t)=-B(t) d \phi(X-d X, t)
$$

which agrees with Eq. (29).

\section{B The differential of the option price}

Let us derive the differential of the call price, $d C$, using either Itô and Stratonovich interpretations. The starting point for both derivations is the replicating portfolio, Eq. (27),

$$
C(X, t)=X(t) \delta_{n}(X, t)+B(t) \phi_{n}(X, t) .
$$


Taking into account the Itô product rule Eq. (11), we have

$$
\begin{aligned}
d C=\left[\delta_{n}(X, t)+d \delta_{n}(X, t)\right] d X & +\left[\phi_{n}(X, t)+d \phi_{n}(X, t)\right] d B \\
& +X(t) d \delta_{n}(X, t)+B(t) d \phi_{n}(X, t),
\end{aligned}
$$

which, after using Eq. (A.3), reads

$$
\begin{aligned}
d C=\delta(X, t) d X+\phi(X, t) d B & +X(t) d \delta_{n}(X, t) \\
& +B(t) d \phi_{n}(X, t)+O\left(d t^{3 / 2}\right),
\end{aligned}
$$

and this agrees with Eq. (28).

Within the Stratonovich calculus, the differential of Eq. (B.1) reads

$$
d C=X_{S}(t) d \delta_{n}+B(t) d \phi_{n}+\delta_{n}\left(X_{S}, t\right) d X+\phi_{n}\left(X_{S}, t\right) d B .
$$

From Eq. (A.1) we have

$$
\delta_{n}\left(X_{S}, t\right)=\delta\left(X_{S}, t\right)-\frac{\partial \delta\left(X_{S}, t\right)}{\partial X_{S}} d X+O\left(d X^{2}\right),
$$

and analogously for $\phi_{n}$. Substituting Eq. (B.3) into Eq. (B.2), and taking into account Eqs. (4)-(5), (10) and (26) we obtain

$$
\begin{aligned}
d C=[X(t) & +d X / 2] d \delta_{n}+B(t) d \phi_{n}+\delta\left(X_{S}, t\right) d X \\
& +\left[r B(t) \phi\left(X_{S}, t\right)-\sigma^{2} X_{S}^{2} \frac{\partial \delta\left(X_{S}, t\right)}{\partial X_{S}}\right] d t+O\left(d t^{3 / 2}\right)
\end{aligned}
$$

But from Eq. (A.1) and the self-financing strategy (A.4), we see that $X(t) d \delta_{n}+$ $B(t) d \phi_{n}=0$. Hence

$$
\begin{aligned}
d C=\frac{1}{2} d X d \delta_{n} & +\delta\left(X_{S}, t\right) d X \\
& +\left[r B(t) \phi\left(X_{S}, t\right)-\sigma^{2} X_{S}^{2} \frac{\partial \delta\left(X_{S}, t\right)}{\partial X_{S}}\right] d t+O\left(d t^{3 / 2}\right)
\end{aligned}
$$

The substitution of the Stratonovich rule Eq. (20),

$$
d \delta_{n}=\frac{\partial \delta_{n}\left(X_{S}, t\right)}{\partial X_{S}} d X+\frac{\partial \delta_{n}\left(X_{S}, t\right)}{\partial t} d t
$$

yields 


$$
\begin{aligned}
d C=\delta\left(X_{S}, t\right) d X & +\left[r B(t) \phi\left(X_{S}, t\right)\right. \\
& \left.-\frac{1}{2} \sigma^{2} X_{S}^{2} \frac{\partial \delta\left(X_{S}, t\right)}{\partial X_{S}}\right] d t+O\left(d t^{3 / 2}\right)
\end{aligned}
$$

where we have taken into account Eq. (4) and the fact that $\partial \delta_{n} / \partial X_{S}=$ $\partial \delta / \partial X_{S}+O\left(d t^{1 / 2}\right)$. Eq. (B.5) agrees with Eq. (38) and the rest of the derivation is identical to that of the main text.

\section{Solution to the Black-Scholes equation}

In this appendix we outline the solution to the Black-Scholes equation (35) under conditions (42)-(44).

We first transform Eq. (35) into a forward parabolic equation with constant coefficients by means of the change of variables

$$
z=\ln \left(x / x_{C}\right), \quad t^{\prime}=T-t
$$

We have

$$
\frac{\partial C}{\partial t^{\prime}}=-r C\left(z, t^{\prime}\right)+\left(r-\frac{1}{2} \sigma^{2}\right) \frac{\partial C}{\partial z}+\frac{1}{2} \sigma^{2} \frac{\partial^{2} C}{\partial z^{2}}
$$

$\left(-\infty<z<\infty, 0<t^{\prime}<T\right)$. Moreover, the definition of a new dependent variable:

$$
u\left(z, t^{\prime}\right)=\exp \left[-\frac{1}{2}\left(1-\frac{2 r}{\sigma^{2}}\right) z+\frac{1}{8} \sigma^{2}\left(1+\frac{2 r}{\sigma^{2}}\right)\left(T-t^{\prime}\right)\right] C\left(z, t^{\prime}\right)
$$

turns Eq. (C.2) into the ordinary diffusion equation in an infinite medium

$$
\frac{\partial u}{\partial t^{\prime}}=\frac{1}{2} \sigma^{2} \frac{\partial^{2} u}{\partial z^{2}}
$$

with a constant diffusion coefficient given by $\sigma^{2} / 2$, and initial condition:

$$
\begin{aligned}
u(z, 0)=x_{C} \exp \left[-\frac{1}{2}(1\right. & \left.-\frac{2 r}{\sigma^{2}}\right) z \\
& \left.+\frac{1}{8} \sigma^{2}\left(1+\frac{2 r}{\sigma^{2}}\right) T\right] \max \left(e^{z}-1,0\right) .
\end{aligned}
$$


The solution of problem (C.4)-(C.5) is standard and reads [31]

$$
u\left(z, t^{\prime}\right)=\frac{1}{\sqrt{2 \pi \sigma^{2} t^{\prime}}} \int_{-\infty}^{\infty} u(y, 0) e^{-(z-y)^{2} / 2 \sigma^{2} t^{\prime}} d y .
$$

If we substitute the initial condition (C.5) into the right hand side of this

equation and undo the changes of variables we finally obtain the Black-Scholes formula Eq. (45).

\section{References}

[1] J.C. Cox, M. Rubinstein, Option Markets, Prentice-Hall, New-Jersey, 1985.

[2] L. Bachelier in: The random character of stock market prices, P.H. Cootner editor, M.I.T. Press, Cambridge, MA, 1964, p.17.

[3] C.W. Smith, J. Financial Econ. 3 (1976) 3-51.

[4] F. Black, M. Scholes, J. Pol. Econ. 81 (1973) 637-659.

[5] R.C. Merton, Bell J. Econ. and Management Sci. 4 (1973) 141-183.

[6] J.P. Bouchaud, M. Potters, Théorie des risques financiers, Aléa-Saclay, Paris, 1997.

[7] B.B. Mandelbrot, Fractals and Scaling in Finance, Springer-Verlag, Berlin, 1997.

[8] B. Arthur, Science 284 (1999) 107-109.

[9] T. Lux, M. Marchesi, Nature 397 (1999) 498-500.

[10] P. Bak, M. Paczuski, M. Shubik, Physica A 246 (1997) 430-453.

[11] E. Fama, J. Business 38 (1965) 34-105.

[12] J.Y. Campbell, A. Lo, A.C. MacKinlay, The Econometrics of Financial Markets, Princeton University Press, Princeton, 1997.

[13] I.I. Gihman, A.V. Skorohod, Stochastic Differential Equations, SpringerVerlag, New York, 1972.

[14] R.C. Merton, J. Financial Econ. 3 (1976) 125-144.

[15] J.C. Cox, S.A. Ross, J. Financial Econ. 3 (1976) 145-166.

[16] K. Itô, On Stochastic Differential Equations, Mem. Am. Math. Soc., 1951, 4, $1-51$.

[17] R.L. Stratonovich, SIAM J. Control 4 (1966) 362-371. 
[18] N.G. Van Kampen, J. Stat. Phys. 24 (1981) 175-187.

[19] K.L. Lindenberg, V. Seshadri, K.E. Shuler, and B.J. West, in: Probabilistic Analysis and Related Topics, A.T. Bharucha-Reid editor, Academic Press, New York, 1983, p.81.

[20] C.W. Gardiner, Handbook of Stochastic Methods, Springer-Verlag, New York, 1983.

[21] J.C. Hull, Options, Futures, and other derivatives, Prentice Hall, New York, 1997.

[22] M. Baxter, A. Rennie, Financial Calculus, Cambridge University Press, Cambridge, 1998.

[23] P. Wilmott, Derivatives, J. Wiley, New York, 1998.

[24] P. Wilmott, J. Dewyne, S. Howison, Option Pricing, Oxford Financial Press, Oxford, 1993.

[25] R.C. Merton, Continous Time Finance, Blackwell, Cambridge, MA, 1992.

[26] M.F. Osborne in: The random character of stock market prices, P.H. Cootner editor, M.I.T. Press, Cambridge, MA, 1964, p.100.

[27] R.C. Merton in: P. Samuelson, SIAM Rev. 15 (1973) 1, p.34.

[28] M. Harrison, S. Pliska, Stoch. Proc. Appl. 11 (1981) 215-260.

[29] It can be easily proved that the net zero investment requirement is equivalent to the absence of arbitrage opportunities, i.e., identical cashflows have identical values.

[30] W.F. Sharpe, J. Finance 19 (1964) 425-442.

[31] H.S. Carslaw, J.C. Jaeger, Conduction of Heat in Solids, Oxford Science Publications, Oxford, 1990. 